\title{
Politique
}

Politique

\section{Charte et société : vers un nouvel ordre politique canadien?}

\section{François Rocher et Daniel Salée}

Numéro 20, automne 1991

URI : https://id.erudit.org/iderudit/040698ar

DOI : https://doi.org/10.7202/040698ar

Aller au sommaire du numéro

Éditeur(s)

Société québécoise de science politique

ISSN

0711-608X (imprimé)

1918-6584 (numérique)

Découvrir la revue

Citer cet article

Rocher, F. \& Salée, D. (1991). Charte et société : vers un nouvel ordre politique canadien? Politique, (20), 35-64. https://doi.org/10.7202/040698ar

\section{Résumé de l'article}

L'enchâssement de la Charte des droits et libertés dans la Constitution canadienne est généralement perçu comme une avancée politique importante dans l'histoire du pays. Presque toutes les analyses s'accordent pour conférer à la Charte des vertus démocratiques qui devraient nécessairement conduire à la mise en place, au Canada, d'un contrat social plus flexible et plus ouvert. Rares sont les analyses qui tentent plutôt d'en évaluer l'impact social réel ou de saisir le sens de l'ordre politique nouveau qu'elle impose inévitablement. Le présent texte veut commencer à rectifier cette lacune en proposant les éléments d'une problématique d'interprétation de la Charte canadienne des droits et libertés à la lumière des mutations sociales et économiques qui, depuis les années 1970, ont conduit à l'instauration d'un nouvel ordre politico-administratif au Canada. Les auteurs soutiennent qu'en insistant sur les droits individuels, la Charte neutralise la mouvance collectiviste et sociale-démocrate instituée par l'État-providence et tend à banaliser le contenu conflictuel des rapports sociaux. Sans le savoir, les formulateurs de la Charte ont peut-être posé, pour l'an 2000, les jalons d'une profonde crise de la gouverne et de la légitimité.
Ce document est protégé par la loi sur le droit d'auteur. L'utilisation des services d’Érudit (y compris la reproduction) est assujettie à sa politique d'utilisation que vous pouvez consulter en ligne.

https://apropos.erudit.org/fr/usagers/politique-dutilisation/ 


\title{
CHARTE ET SOCIÉTÉ : VERS UN NOUVEL ORDRE POLITIQUE CANADIEN?
}

\author{
François Rocher \\ Université Carleton \\ Daniel Salée \\ Université Concordia
}

L'enchâssement de la Charte des droits et libertés dans la Constitution canadienne est généralement perçu comme une avancée politique importante dans l'histoire du pays. Presque toutes les analyses s'accordent pour conférer a la Charte des vertus démocratiques qui devraient nécessairement conduire à la mise en place, au Canada, d'un contrat social plus flexible et plus ouvert. Rares sont les analyses qui tentent plutot $d^{\prime}$ 'en évaluer l'impact social réel ou de saisir le sens de l'ordre politique nouveau qu'elle impose inévitablement. Le présent texte veut commencer à rectifier cette lacune en proposant les éléments d'une problématique d'interprétation de la Charte canadienne des droits et libertés a la lumière des mutations sociales et économiques qui, depuis les années 1970, ont conduit a l'instauration d'un nouvel ordre politico-administratif au Canada. Les auteurs soutiennent qu'en insistant sur les droits individuels, la Charte neutralise la mouvance collectiviste et sociale-démocrate instituée par l'État-providence et tend à banaliser le contenu conflictuel des rapports sociaux. Sans le savoir, les formulateurs de la Charte ont peut-être posé, pour l'an 2000, les jalons d'une profonde crise de la gouverne et de la légitimité.

D'un point de vue strictement formel, l'enchåssement, en 1982, de la Charte des droits et libertés dans la Constitution canadienne représente un gain sur la situation qui prévalait jusqu'alors. La Déclaration des droits ratifiée en 1960 par le gouvernement Diefenbaker ne s'adressait qu'aux institutions fédérales et n'avait qu'un caractère statutaire équivalent a celui de tout autre acte législatif du Parlement canadien. Au mieux, on ne pouvait que lui reconnaître une portée morale et souhaiter qu'elle fût utilisée de manière qu'aucune loi ne soit 
appliquée ou interprétée en contrevenant aux droits et libertés qu'elle formulait (Tarnopolsky, 1983, p. 261-264).

Armés de directives aussi peu explicites, les juges de la Cour suprême hésitèrent toujours à donner préséance à la Déclaration des droits et s'en tinrent, à une exception près, au principe de suprématie législative'. La Déclaration des droits $n^{\prime}$ offrait pas à leurs yeux un mandat constitutionnel suffisamment clair, qui leur eût permis de rendre des décisions limitant ou remettant en question la souveraineté traditionnelle du Parlement (Greene, 1989, p. 23-29).

L'enchâssement de la Charte dans la Constitution a conféré à celle-ci une supériorité juridique sur toute législation et réglementation émanant non seulement du gouvernement fédéral, mais aussi de tous les autres paliers gouvernementaux et administratifs. En théorie, elle permet une protection beaucoup plus étendue contre des abus de pouvoir politicoadministratifs et des actes discriminatoires éventuels de l'État ou de toute autre institution socio-économique (Williams, 1985, p. 121). Elle recèle dans son principe la promesse d'une société plus juste, plus égalitaire et plus respectueuse des caractéristiques et prétentions individuelles.

II s'en trouvera bien peu pour affirmer que la Charte constitue un document irréprochable. Bon nombre d'observateurs et $d$ 'analystes de l'objet constitutionnel n'ont pas manqué d'en relever les imperfections et les limites (Banting et Simeon, 1983; Russell, 1985, 1990; Milne, 1990). Néanmoins, on semble généralement croire que la Charte représente une avancée politique importante. Pour Roy Romanow, acteur de premier plan dans les négociations

1. L'arrêt Drybones de 1969 est le seul dans lequel une majorité de juges de la Cour suprême ait donné préséance a la Déclaration des droits de manière à rendre inopérant un aspect d'une autre loi. Dans ce cas-ci, les magistrats reconnurent qu'une section de la Loi des Indiens, qui imposait des sanctions pénales plus sévères aux autochtones qu'à la population en général dans les cas d'ébriété sur la voie publique, constituait de la discrimination raciale en vertu du principe d'égalité devant la loi contenu dans la Déclaration des droits. Dans toutes les autres causes, les juges qui s'inspirèrent de la Déclaration furent minoritaires. 
intergouvernementales préalables au pacte de 1982 , elle permet d'accroitre la participation politique et de faire obstacle à la tyrannie de la majorité (Romanow, Whyte et Leeson, 1984, p. xx). Dans le même esprit, David Elkins s'est réjoui de ce que la Constitution canadienne se distinguât de la Constitution américaine et qu'elle allât même plus loin à certains égards. II soutient que la reconnaissance de droits et avantages collectifs, de même que l'existence de droits négatifs et la possibilité d'un recours à la clause nonobstant, contribue au respect des particularismes communautaires et, ce faisant, à la préservation de l'intégrité des individus qui se réclament de ces particularismes. En ce sens, la Constitution canadienne est unique dans un monde où les différences culturelles, ethniques, raciales et idéologiques sont souvent prises à partie et même violemment réprimées (Elkins, 1989).

David Beatty, juriste de l'université de Toronto, va plus loin et estime que le pouvoir accordé par la Charte à l'instance judiciaire en tant que gardienne des droits et libertés assure aux défavorisés de la société une plus grande participation politique et une meilleure protection. C'est toute la qualité du contenu démocratique de la "gouverne» qui s'en trouve améliorée. Le processus de révision judiciaire, auquel la Charte permet de soumettre les lois, oblige les agents sociaux qui sont en opposition à avoir un dialogue neutre et non partisan. Il force les bénéficiaires d'un avantage législatif à en justifier le bien-fondé auprès de tous les autres qui n'en profiteraient pas au même degré et qui considéreraient que leurs prérogatives constitutionnelles sont lésées (Beatty, 1987).

D'autres, enfin, se montrent plus nuancés. Si la Charte n'a pas produit tous les résultats escomptés, ils reconnaissent volontiers qu'elle contient tout de même un potentiel démocratique important pouvant aisément être mis à profit pour la promotion des droits humains et des libertés individuelles et, par conséquent, pour la mise en place d'un contrat social et politique plus flexible et plus ouvert (Greene, 1989, p. 222 228).

Lorsqu'elles parviennent à déborder les limites d'un légalisme formel et technique, les abondantes études sur la 


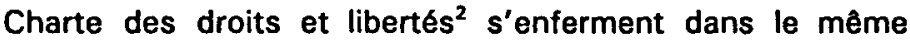
discours convenu : I'enchåssement des droits et libertés dans la Constitution a ouvert une nouvelle ère politique au Canada, et il n'en tient qu'à la population elle-même d'en faire un instrument de développement social. II $n^{\prime} y$ aurait de limites à un tel usage que celles que la population voudra bien se donner. Études basées sur de bonnes intentions, donc, qui reflètent plus souvent les à priori moraux et politiques des auteurs. Rares sont les analyses qui tentent plutôt d'évaluer l'impact social réel de la Charte ou de saisir le sens de celle-ci en regard de l'ordre politique nouveau qu'elle impose inévitablement.

Pourtant, les années 80 ont amorcé une période de transition qui se caractérise par des tentatives de plus en plus précises et répétées pour redéfinir les rapports de force, le réaménagement du tissu socio-économique et la restructuration de l'ordre politico-administratif. Les années qui s'en viennent seront déterminantes. Le Canada du XXI' siècle participera en quelque sorte de ce que la réorganisation globale de la société de cette fin de siècle aura ou n'aura pas été. La Charte des droits et libertés enchâssée dans l'Acte constitutionnel de 1982 constitue la pièce maîtresse de cette réorganisation. Elle sera donc examinée de près ici, non pas tant dans son contenu formel ${ }^{3}$ que dans ses fondements philosophiques et son impact sociopolitique réel.

Le propos s'attachera à démontrer jusqu'à quel point l'ordre politique articulé par la Charte représente en fait une réponse institutionnelle à la crise de la société et de l'économie canadiennes. En insistant sur les droits individuels, la Charte neutralise la mouvance collectiviste et sociale-démocrate créée par l'État-providence et elle tend à banaliser le contenu conflictuel des rapports sociaux. En procédant ainsi

2. Voir l'impressionnante bibliographie détaillée que I'on trouve dans Beaudoin et Ratushny (1989, p. 843-923).

3. L'exercice a déjà été accompli par d'autres (Milne, 1989; Greene, 1989; Beaudoin et Ratushny, 1989). 
à une telle déconstruction de la société civile, la Charte témoigne paradoxalement de l'essoufflement et des contradictions du modèle libéral-démocratique au Canada. Sans le savoir, les formulateurs de la Charte ont peut-être posé pour l'an $\mathbf{2 0 0 0}$ les jalons d'une profonde crise de "gouverne" et de légitimité.

Le présent texte propose en quelque sorte les éléments d'une problématique d'interprétation de la Charte canadienne, a la lumière des mutations sociales et économiques qui ont conduit à un nouvel ordre politico-administratif au Canada. L'intelligibilité de la Charte ne peut pas être exclusivement fondée sur les projets politiques mis de l'avant par les acteurs, Trudeau en tête. Si la Charte et la Constitution qui la porte ont pu recevoir un accueil favorable de la majorité de la population canadienne, c'est notamment parce qu'elles $s^{\prime}$ inscrivaient dans une mouvance de société dont il importe maintenant de saisir les contours.

\section{L'hégémonie en péril :}

\section{la Charte et la gestion de la crise}

II serait naîf de faire abstraction du contexte éminemment politique et des enjeux politiciens qui ont marqué le processus d'enchâssement de la Charte. Dans l'esprit de ses formulateurs, l'enchâssement devait aussi servir d'expédient politique pour neutraliser les menaces croissantes faites à la légitimité et à l'intégrité de l'État canadien (Mandel, 1989, p. 32). Mais, au-delà des impératifs politiques, la Charte fut mise en place dans le contexte d'une crise structurelle de l'économie qui remettait en question la capacité d'intervention de l'État canadien et qui s'inscrivait dans une dynamique de consolidation du pouvoir exercé par le niveau central de l'État. La Charte a contribué à modifier la nature des rapports politicoéconomiques découlant de la forme fédérative de l'état. Aussi, malgré les aspirations humanistes et égalitaristes du libellé, l'adoption de la Charte participe d'une double stratégie : renforcement du palier central de l'État canadien et reviviscence du sentiment $d$ 'appartenance nationale. Sous couvert d'idéaux grandioses et moralement incontestables, 
l'enchåssement de la Charte dans la Constitution devait fonctionner comme outil politique grâce auquel ses initiateurs espéraient contrer les forces provincialistes et régionalistes montantes, et créer les conditions d'émergence d'une identité et d'alliances nationales. Dénominateur commun par excellence, la cause des droits de la personne rallierait le pays tout entier sous l'égide d'un gouvernement central fort (Knopff et Morton, 1985).

La Charte s'inscrit dans une profonde crise de légitimité du contrat social établi après la Seconde Guerre mondiale. C'est dans la mouvance historique de la lutte des classes, des affrontements entre dominants et dominés et des stratégies qui leur sont propres que la Charte révèle sa logique politique fondamentale. Au cours des années 70 , le Canada a connu une crise économique qui a mis fin a la période quasi ininterrompue de prospérité ayant marqué l'après-guerre. II ne s'agissait pas là d'une simple crise cyclique. Les conséquences particulières de cette dégradation de l'économie (maintien des taux de chômage et d'inflation à des niveaux relativement élevés; accroissement du déficit financier de l'État; et faiblesse continue de la productivité) indiquaient au contraire une crise profonde du système d'accumulation capitaliste et du rapport salarial (Boismenu, 1990).

Depuis la récession de la fin des années 70 et du début des annés 80 , la reproduction du capitalisme au Canada doit faire face non seulement aux pressions économiques domestiques, mais aussi aux contraintes exercées par la tendance irrévocable à la mondialisation de l'économie. Les nombreux changements au sein de l'économie internationale ont touché de façon différente les pays industrialisés. Le Canada n'a pas échappé à ces importantes mutations et a dô réévaluer la place qu'il occupe dans la division internationale du travail. Producteur de matières premières et de produits finis, il se situe a la jonction des courants qui bouleversent les flux économiques et commerciaux. Le Canada se caractérise notamment par la très grande ouverture de son économie. II est donc particulièrement sensible aux changements qui affectent le marché international. Transformations qui se sont traduites par un déclin de sa position relative dans le com- 
merce mondial (Gouvernement du Canada, 1983, p. 24). Cette décroissance s'est surtout manifestée dans les branches a haute intensité du domaine technologique. La part du Canada sur les marchés mondiaux s'est maintenue en revanche dans les branches à moyenne et faible intensités. Dans ce contexte, la capacité d'adaptation du Canada n'est guère prometteuse (Finlayson, 1985, p. 64), d'autant que l'évolution de ses relations commerciales depuis trois décennies démontre sa difficulté à s'affranchir d'un double problème. D'une part, le contrôle étranger sur certains secteurs économiques empêtre le commerce international de produits manufacturés dans des liens de dépendance qui limitent les possibilités d'intervention gouvernementale. D'autre part, la faible proportion qu'occupent les produits finis dans les exportations condamne la structure industrielle du pays à une anémie chronique (Houle, 1987).

L'inévitable intégration de l'économie canadienne au marché mondial soumet à des contraintes importantes les rapports entre le capital national et le capital étranger. Pour réussir à faire face aux nouveaux défis que posent à la fois la crise du capitalisme domestique et l'internationalisation du capital, la bourgeoisie canadienne a été forcée, au début des années 80 , de réorganiser complètement l'économie.

Mais la chose n'est pas simple. Pour être effectués de manière cohérente et efficace, les ajustements que suppose une restructuration de l'économie exigent l'intermédiaire de l'État. Plus que jamais, l'État est au cœur de la réorganisation des rapports capitalistes et en constitue même un des enjeux majeurs. Par-delà sa crise financière, il doit redéfinir son rôle en fonction des besoins du capital, de manière à favoriser une privatisation accrue des activités du secteur public et à se soumettre encore plus explicitement à la logique de l'accumulation (Wolfe, 1984, 1989). L'État doit donc soutenir encore plus directement les entreprises les mieux placées pour faire face à la concurrence internationale, et ainsi relancer l'économie domestique.

Le mode d'organisation de l'État complique la redéfinition de son rôle. La dynamique fédérale de l'État est ainsi faite que tous les niveaux de gouvernement ne privilégient pas 
nécessairement des stratégies de gestion de crise identiques ni même complémentaires. Cependant, certaines stratégies se recoupent parfois, notamment en ce qui a trait à la réduction des dépenses publiques et aux restrictions des programmes sociaux. L'expérience récente du fédéralisme canadien démontre en fait que chaque niveau de gouvernement a plutôt tendance à adopter des stratégies qui renvoient consciemment le fardeau des problèmes à l'autre palier. Depuis la révision des accords fiscaux de 1982, le gouvernement fédéral n'a pas cessé de réduire les paiements de transfert aux provinces pour améliorer sa propre position financière. L'équilibre entre la capacité fiscale et les responsabilités de dépenses a été au centre des négociations sur les arrangements fiscaux. Le gouvernement central veut contrer la tendance, endémique depuis la Seconde Guerre mondiale, à la diminution relative de la part fédérale des recettes publiques. A partir de cette époque, les provinces ont accru leur capacité fiscale et peuvent plus aisément promouvoir des objectifs allant à l'encontre des plans d'union économique, pourtant si fondamentaux pour la restructuration de l'économie nationale.

Dans ce contexte particulièrement chargé, le gouvernement central doit se donner une stratégie à double action. D'une part, il faudrait que cette stratégie élargisse les possibilités d'intervention du niveau central; celui-ci coordonnerait les politiques de stabilisation et de développement. D'autre part, elle réduirait le plus possible la portée des interventions provinciales pouvant avoir des effets négatifs sur l'économie des autres provinces. L'intégrité de l'État canadien tient en somme dans sa capacité à harmoniser les politiques économiques fédérales et provinciales. Au cours des années 80 , cette capacité a souvent été en butte à l'hostilité des provinces. En cherchant plutôt à maximiser leurs revenus, à augmenter le nombre d'emplois et a favoriser la croissance de la population sur leurs territoires respectifs (Maxwell et Pestieau, 1980, p. 84), les provinces ont souvent offert une stratégie de gestion de crise qui divergeait de celle du palier central de l'État et menaçait en partie la réalisation de l'intégration économique. A la dynamique de fragmentation, le gouverne- 
ment fédéral a opposé avec vigueur une logique d'unification, qui pose la redéfinition des relations fédérales/provinciales et des rapports centre/périphérie, redéfinition qu'il voudrait favorable au palier central de l'État.

A un niveau global, donc, le succès de la restructuration de l'économie, engendrée par la crise du régime d'accumulation, dépend de la remise en cause des lieux d'exercice du pouvoir. Mais, à un niveau plus spécifique, il passe par la révision à la baisse de la norme salariale et des avantages qui limitent les prérogatives du capital au sein du processus de travail. Enfin, il passe par la compression des dépenses étatiques et par la rationalisation - voire l'élimination - des programmes sociaux. Autrement dit, le succès de la restructuration tient dans la remise en question des quelques gains historiques consentis aux régions et groupes dominés ou défavorisés depuis la fin de la Seconde Guerre mondiale. C'est tout l'équilibre des rapports de force socio-économiques qui est en jeu. Le libre marché, la libre entreprise, I'union économique et l'individualisation du social s'imposent désormais comme les normes dominantes d'interaction en société et renvoient au statu quo prékeynésien.

Tant que les conditions d'une organisation stable et durable des rapports sociaux (combinaison d'une forte productivité et de salaires relativement élevés) se sont maintenues, les rapports de force socio-économiques ont pu jouer quelque peu en faveur des groupes historiquement dominés ou défavorisés. La négociation collective est devenue un mécanisme institutionnel de relations entre le capital et le travail, qui est largement accepté. Assurant le lien entre la production de masse et la consommation de masse, la négociation a profité à la fois à la classe ouvrière et aux capitalistes. De même, il est maintenant admis que, par son rôle économique régulateur, l'État-providence a répondu tout autant aux aspirations collectivistes et socialisantes de la société civile qu'aux besoins particuliers du capital.

$A$ travers des engagements financiers considérables, l'État a essayé de répondre à la diversité des revendications émanant de la société civile. Bien que souvent insuffisants, la plupart des programmes d'assistance sociale, d'aide à 
l'emploi, d'action positive ou de développement régional ont été instaurés pour aplanir quelque peu les disparités de statut social et économique, et pour répondre a des réalités de société nouvelles et désormais incontournables. D'une certaine manière, ces programmes reconnaissaient les potentialités conflictuelles de la société civile et y proposaient des solutions.

Tout cela a été possible jusqu'à la fin des années 70 environ, dans la mesure où, précisément, les conditions économiques donnaient une certaine stabilité aux rapports de force qui assurent le lien entre la production de masse et la consommation de masse. Mais, depuis le début des années 80 , ces conditions n'existent plus. Depuis lors, le capital canadien est contraint de répondre aux pressions croissantes de la concurrence internationale et de l'internationalisation de la production. Les avantages consentis par le passé aux groupes dominés et défavorisés sont désormais perçus comme un frein à la restructuration. Le déficit budgétaire de l'État, bien qu'en partie créé par l'aide financière aux entreprises, est vu comme un obstacle a la remise en forme de l'économie (Langille, 1987).

L'offensive du capital contre le travail est réelle (Drache et Glasbeek, 1990). Bien qu'elle porte à priori sur les mécanismes institutionnels qui ont permis l'élargissement des droits de la classe ouvrière, elle entraîne dans sa foulée une série d'attaques contre les avantages sociaux et économiques que les groupes dominés avaient acquis depuis un certain temps. Elle empêche aussi l'extension de ces mêmes avantages. En témoignent la réforme de l'assurance-chômage, la réforme du bien-être social au Québec et la recriminalisation très probable de l'avortement. Mais ce sont surtout les groupes les plus démunis, victimes historiques des inégalités systémiques, qui font les frais de la restructuration : les femmes monoparentales, les jeunes cantonnés dans les emplois précaires, les minorités visibles, les travailleuses et les travailleurs sousscolarisés.

Que vient faire la Charte dans la restructuration et la gestion de la crise? II faut voir qu'elle pose les paramètres $d^{\prime} u n$ nouveau mode d'intervention et $d^{\prime}$ 'interaction politiques. 
Mais il faut voir surtout qu'elle s'insère dans la crise structurelle de l'économie canadienne en tant que stratégie justificatrice du changement de société que les impératifs de la restructuration vont impliquer. La section qui suit tentera de démontrer que la Charte sert de fer de lance aux classes et groupes dominants de la société canadienne dans la lutte contre la menace à leur hégémonie rendue fragile.

\section{Charte et société : à la recherche du sens ${ }^{4}$}

A un premier niveau d'effets, l'enchåssement de la Charte dans la Constitution doit accentuer l'unité/identité nationale et réaffirmer le centre comme maître-d'œuvre politique de l'ensemble du pays au détriment des régions (Knopff et Morton, 1985; Jenson, 1989). Elle s'inscrit dans un ensemble de politiques concomitantes, inaugurées au début des années 80 et qui visent entre autres à rapatrier au gouvernement fédéral le contrôle du développement économique. Mais, à un autre niveau, plus fondamental encore, la Charte impose de nouveau le discours de l'égalitarisme et de l'individualisme libéral au moment même où la dominance du marché et l'individualisme socio-économique sont présentés comme les seules solutions plausibles à la crise. La Charte offre une norme légale légitime - et incontournable parce que légale qui justifie les attaques contre les excès du collectif, du politique et de la société civile, de même que contre un modus vivendi social devenu coûteux et donc inacceptable aux classes et groupes dominants. En effet, en autorisant les tribunaux a statuer sur le bien-fondé des législations, la magistrature se voit investie des rôles de décideur ultime et de formulateur de politiques publiques. Dans la mesure où les gouvernants renvoient volontiers au pouvoir judiciaire toute question délicate qui relève de la Charte, le système politique se laisse glisser, sous l'égide de la Cour suprême, vers la centralisation du processus d'élaboration de politiques en

4. Cette section s'inspire des travaux de Mandel, 1989, de Petter, 1986, 1987, 1989, de Brodsky et Day, 1989, et de Hutchinson et Petter, 1988. 
matière de justice sociale et de droits linguistiques des minorités (Russell, 1982; Morton, 1987).

Déçus des canaux politiques et bureaucratiques traditionnels, les groupes de pression se réclament davantage maintenant de la Charte et se tournent plus fréquemment vers les tribunaux dans la poursuite de leurs objectifs politiques. De même, les domaines législatifs de juridiction provinciale s'étiolent sous l'imposition de critères nationaux rendus uniformes en vertu de jugements émis par la Cour suprême et portant sur la Charte.

Désormais, les juges "font" la loi en même temps qu'ils l'appliquent. Le principe, fondamental en démocratie libérale, qui veut que seuls les élus soient habilités à légiférer, s'en trouve ainsi renversé (Russell, 1987). La Charte offre une justification commode au recours systématique à la justice, chaque fois qu'un objectif politique est trop complexe ou trop difficile à atteindre par les voies représentatives et démocratiques normales. L'accentuation du pouvoir judiciaire s'accomplit donc en opposition directe avec les formes populaires de pouvoir et à l'organisation de la justice (Mandel, 1989, p. 32).

En judiciarisant le politique, la Charte remplace la norme politique par la norme légale. Le caractère technique de celleci recouvre les problèmes socio-économiques d'un voile d'abstractions destiné à déguiser et à banaliser le contenu politique des choix de société. La prédominance du droit sur le politique interdit, au nom de l'universalité et de l'authenticité des valeurs contenues dans la Charte, «toute polémique publique portant non pas sur ces principes généraux, effectivement admis, mais bien sur la manière dont ils sont institutionnalisés, sur la gestion qui en est faite [...] comme si s'interroger sur le mode d'emploi des droits de l'homme revenait en quelque sorte à s'opposer à l'idée même de droits fondamentauX» (Vandycke, 1986, p. 140). Bref, en bout de piste, l'enchâssement des droits et libertés dans la Constitution semble devoir "conduire à un rétrécissement significatif de l'espace public à l'intérieur duquel s'exerce le débat démocratique entre les citoyens" (Vandycke, 1986, p. 139).

Mais la portée véritable de la Charte ne réside pas tant dans la création de nouveaux droits que dans la façon dont les 
droits sont gérés. C'est alors tout le système des rapports de pouvoir au sein de la société canadienne qui s'en est trouvé affecté. C'est là un aspect que négligent certains critiques de la judiciarisation du politique. Ils oublient que la judiciarisation est beaucoup plus qu'un simple transfert d'instances (du législatif au judiciaire) et que cela fait partie d'une transformation du politique, quelle que soit l'instance (Mandel, 1989, p. 71). Dans les faits, l'application de la Charte a contribué au renforcement des mécanismes institutionnels d'exercice du pouvoir socio-économique au profit des classes et groupes qui en ont toujours le plus directement bénéficié. En dépit de sa générosité apparente, la Charte n'a pas été conçue pour faire pencher la balance du pouvoir socio-politique en faveur des groupes et individus qui en ont été exclus historiquement. Elle contribue au contraire à amenuiser la menace qu'ils représentent pour l'ordre établi.

Tout comme les autres sociétés occidentales industrialisées, le Canada est affligé d'inégalités socio-économiques qui, malgré la possibilité théorique d'en sortir et l'atténuation idéologique de leur réalité, sont pratiquement érigées en système (Hunter, 1986). Inégalités de traitement fondées sur l'origine ethnique, l'origine sociale et le sexe; inégalités régionales; inégalités d'accès aux lieux d'exercice du pouvoir; inégalités économiques : toutes tiennent à des pratiques socioculturelles profondément enracinées et historiquement déterminées. Ces inégalités s'objectivent dans des rapports de force éminemment politiques qui ont constamment des répercussions sur le contrat social conclu entre dominants et dominés, privilégiés et défavorisés, puissants et vulnérables. Elles s'expriment dans un code d'interrelation sociale, lequel profite à une minorité de classes ou groupes dominants qui n'ont généralement aucun avantage à en voir les paramètres modifiés.

Plusieurs fondent énormément d'espoir dans ce qu'ils croient être le potentiel distributif de la Charte. Ils y voient l'instrument $d$ 'une plus grande justice sociale, un instrument qui donne à tous sans prendre a personne. Ils $y$ voient un modèle d'égalité formelle offrant les mêmes avantages à tous 
et a toutes, indépendamment de leur condition sociale et économique.

Mais les obstacles à une égalité véritable sont nombreux. La Charte stipule que la gestion de l'application des droits qu'elle garantit relève, en dernier ressort, des tribunaux. Or, dans les faits, les tribunaux ne sont pas toujours des plus accessibles. Le processus légal est souvent long et complexe; le jargon juridique est difficile, voire impossible, à percer pour le profane; le formalisme et le caractère antagonique des procédures sont intimidants. Enfin, la structure hiérarchisée et les procédures d'appel du système judiciaire canadien obligent parfois à gravir de nombreux paliers pour accéder à la Cour suprême et obtenir une décision ultime et irrévocable. A elles seules, ces barrières sont suffisantes pour décourager la plupart des citoyens et citoyennes de se réclamer de la Charte pour faire valoir leurs droits.

A cela s'ajoutent, il va sans dire, les coûts prohibitifs liés à toute action judiciaire, coûts que seuls les sociétés, les organismes publics ou des individus bien nantis peuvent généralement assumer ${ }^{5}$. Les causes criminelles constituent, a toutes fins utiles, l'unique contexte dans lequel un individu peut se réclamer de la Charte et recevoir l'appui financier de l'État ${ }^{\circ}$. C'est, à n'en pas douter, un gain important. La

5. A titre d'exemple, la société Southam Newspapers a déboursé $200000 \$$ en frais judiciaires après une année et demie de procès pour contester, en vertu de l'article 8 de la Charte, une perquisition faite dans ses bureaux par des enquêteurs de la brigade anti-collusion. La National Citizens Coalition, quant à elle, a déboursé plus de 400000 \$ pour appuyer la requête de Merv Lavigne qui s'était insurgé contre l'utilisation des redevances syndicales a des fins politiques, en alléguant qu'une telle pratique contrevenait à sa liberté d'association garantie par la Charte. (Mandel, 1989. p. 43; Petter, 1986, p. 481).

6. II faut noter, en toute justice, que le gouvernement fédéral a mis en place un fonds de soutien - pour les causes ximportantes" seulement - en matière d'égalité et de droits linguistiques. De même, certaines provinces ont mis à la disposition du public des ressources monétaires et techniques ad hoc pour aider a la conduite de causes non criminelles qui pourraient relever de la Charte. Toutefois, jamais encore il ne s'est agi de politiques systématiques et universelles (Petter, 1986, p. 481). En créant le Programme de contestation judiciaire en 1985, le gouvernement fédéral a établi une mesure pour 
plupart des causes invoquant la Charte ont été jusqu'à maintenant de nature criminelle; où, la majorité des gens qui font face a des accusations criminelles sont eux-mêmes démunis (Petter, 1986, p. 481).

II faut admettre toutefois que les questions de droits soulevées par les procédures criminelles ont un impact social et économique marginal. Les droits dont se réclament les individus dans un procès inscrit au criminel sont le plus souvent des droits légaux, de nature procédurale et liés à l'exécution de la justice. Ce sont, par exemple, la protection contre l'emprisonnement arbitraire ou des perquisitions injustifiées, le droit à un procès dans des limites de temps raisonnables, etc. Leur portée dépasse rarement le contexte criminel. Leur application en bonne et due forme protège surtout contre des pratiques abusives des pouvoirs policier et pénal, ce qui, bien sûr, n'est pas négligeable.

Mais la Charte ne protège pas que les droits légaux. La liberté d'expression, la liberté d'association et le droit à l'égalité sont des droits plus expressément politiques dont l'application doit, en principe, procurer une plus grande équité socio-économique et un meilleur équilibre dans les rapports de force au sein de la société.

Les droits à l'égalité, en particulier, pourraient occuper une place centrale dans une éventuelle révision des rapports de force. Cependant, pour atteindre un tel objectif et déployer ces droits dans toute leur efficacité propre, leur application doit déborder leur portée individuelle étroite : elle doit s'adres-

améliorer l'accès aux tribunaux des personnes qui invoquent le droit à l'égalité. Ce programme, administré par le Conseil canadien de développement social, permet aux parties qui, dans une affaire relative à l'égalité, contestent une loi ou un programme fédéral de réclamer par juridiction jusqu'a 35000 \$ pour les frais d'avocats, de recherche et pour les dépenses générales. Le programme devait se terminer en mars 1990, mais it a récemment été reconduit pour une autre période de cinq ans. $D^{\prime}$ après les critiques, il présente cependant trois lacunes graves : les fonds sont offerts uniquement pour les contestations de lois et de programmes fédéraux; un montant de $35000 \$$ par juridiction pour toutes les dépenses est insuffisant pour suivre tout le déroulement d'affaires complexes; les fonds peuvent être utilisés uniquement pour les honoraires des avocats et les recherches relatives à la procédure visée (Brodsky et Day, 1989, p. 146-149). 
ser à l'ensemble des groupes depuis longtemps défavorisés et privés de pouvoir. Bref, elle doit s'adresser à la source systémique de l'inégalité.

Pour bien préparer une cause touchant les droits à l'égalité et en espérer des retombées positives, qu'elle concerne un individu ou un groupe historiquement victime d'inégalité, il faut une expertise qui dépasse la seule compétence juridique. Mais cette expertise est coûteuse, longue à obtenir, et nécessite un appareillage institutionnel et organisationnel souvent déficient ou inexistant chez les groupes et individus défavorisés (Brodsky et Day, 1989).

Lorsqu'ils sont invoqués devant les tribunaux, les droits à l'égalité ne reçoivent pas un appui financier systématique de l'État. Pourtant, une application large de ces droits contribuerait à un meilleur équilibre des forces sociales. Avec le résultat qu'un nombre minime d'actions visant à corriger des situations d'inégalité ou de discrimination systémique ont été intentées jusqu'à maintenant (moins de $3 \%$ de toutes les causes où l'égalité a été invoquée). Dans la majorité des cas où l'égalité a été partie du litige, ou bien l'action avait été intentée par des représentants de groupes traditionnellement dominants (trois fois plus d'hommes que de femmes) qui s'attaquaient à des avantages législatifs acquis par des groupes dominés ou défavorisés, ou bien la référence à l'égalité était tout à fait accessoire et n'avait que peu ou pas d'impact sur la lutte contre l'inégalité systémique et la modification des rapports de force (Brodsky et Day, 1989).

En réalité, la judiciarisation de la gestion des droits à caractère sociopolitique implique un déni de justice aux personnes qui n'ont pas les ressources financières suffisantes pour porter devant les tribunaux des causes de nature non criminelle. En prenant acte de cet état de fait, il ne s'agit pas de revendiquer le secours financier de l'État pour trouver auprès des tribunaux des solutions à des problèmes intrinsèquement politiques. II s'agit plutôt de montrer que la gestion judiciaire des droits impose des limites importantes a leur application égalitaire, à une application susceptible de modifier tant soit peu la configuration des rapports de force socioéconomiques. 
Les difficultés d'accès au pouvoir judiciaire sont un aspect fondamental de cette improbable modification. Dans la mesure où les groupes sociaux historiquement dominants continueront, par le biais systémique qui les avantage, a profiter d'un accès privilégié aux tribunaux, les juges canadiens n'auront que ces modèles de référence. Ils ne pourront être confrontés à des modèles d'égalité qui pourraient avantager les groupes dominés. Dans l'état actuel des choses, urien ne permet de garantir que les tribunaux feront des garanties à l'égalité une application vivante et concrète [...]; si les tribunaux $n$ 'entendent que les interprétations des procureurs de la Couronne, des avocats de la défense et des groupes professionnels, leur optique sera nécessairement très limitée» (Brodsky et Day, 1989, p. 143).

Dans un contexte de judiciarisation poussée du politique, l'amorce éventuelle d'une transformation de l'ordre sociopolitique en faveur des dominés et des défavorisés dépend presque entièrement de la façon dont les tribunaux interprètent les garanties à l'égalité contenues dans la Charte. Les études sur la question affirment généralement que, jusqu'à maintenant, rien ne permet d'entretenir de l'espoir à cet égard.

Tout comme les autres déclarations de droits occidentales, la Charte canadienne des droits et libertés trouve sa cohérence et sa légitimité dans les valeurs et présupposés de la pensée libérale classique. Les droits inscrits dans la Charte reposent sur la conviction que les obstacles à la liberté ne sont pas les disparités socio-économiques entre riches et pauvres ou la concentration du pouvoir dans les mains de quelques privilégiés, mais bien l'État. Selon la théorie libérale des droits, chaque individu existe dans une sphère prépolitique, voire apolitique, d'autonomie et de liberté pures, qui ne dépend en aucune manière de l'État. L'individu constitue une entité indépendante qui interagit avec les autres dans le seul but de satisfaire ses besoins et préférences. En ce sens donc, l'objectif fondamental d'une Charte libérale des droits est de gérer la frontière qui sépare à la fois le collectif de l'individuel et le politique de la sphère prépolitique. En d'autres mots, l'objectif est d'empêcher l'empiètement de l'État sur le domaine naturel de la liberté individuelle. 
On ne trouvera pas dans la Charte de dispositions visant a protéger réellement le droit au travail, à une habitation décente, ou à des services sociaux. Les droits spécifiés dans la Charte sont presque essentiellement de nature négative : ils protègent les individus contre l'ingérence ou le contrôle de l'État. L'interprétation qu'en a déjà donnée le juge en chef Dickson, de la Cour suprême, ne laisse planer à ce propos aucune équivoque : la Charte, croit-il, «is intended to constrain governmental action inconsistent with those rights and freedoms (enshrined within it); it is not in itself an authorization for governmental action" (cité dans Petter, 1989, p. 152).

Voila, en quelque sorte, le fin mot de l'histoire. Certes, il faut reconnaître que la Charte articule aussi certains droits collectifs : les droits linguistiques, la protection des programmes d'action positive, la préservation des droits des autochtones et de l'école confessionnelle en sont les meilleurs exemples (Monahan, 1987). II n'empêche que, à l'exception des droits linguistiques, les préoccupations qui ont trait aux droits collectifs dans la Charte n'ont de sens qu'en ce qu'elles nuancent ou limitent certaines garanties; elles ne modifient en rien le fait que l'individualisme libéral demeure le principe moteur de la Charte. La Cour suprême a d'ailleurs indiqué qu'elle considerait les droits collectifs comme des droits "politiques" bénéficiant d'une plus faible protection constitutionnelle que les droits de principe contenus dans la Charte (Monahan et Petter, 1987).

Par sa philosophie fondamentale, la Charte n'offre pas les moyens de modifier substantiellement le mode dominant de répartition du pouvoir et de la richesse au sein de la société canadienne. II est fondé, par définition, sur l'initiative individuelle et non sur l'action étatique. Le légalisme étroit auquel la Charte ramène toute question de droits, de même que sa négation implicite de la sphère publique comme lieu valide d'intervention, condamne à un échec presque certain toute atteinte à la conception individualiste du social, toute action qui s'inscrirait en dehors du cadre de la stricte égalité formelle des individus. 
Le droit à l'égalité garanti par la Charte ne permet pas la mise en place d'un contexte d'égalité sociale. II assure tout au plus l'égalité de traitement par les instances législatives et exécutives de l'État. Les critères d'égalité seront aussi bien satisfaits par l'action que par l'inaction législative, dans la mesure où chaque membre de la société en est affecté de la même manière. Rien de surprenant, dès lors, à ce que le recours à l'égalité formelle et à son pendant, le critère de situation identique, ait entraîné le retrait d'avantages législatifs à des groupes défavorisés'. Plutôt que de contribuer à l'instauration d'une justice véritable, l'égalité perd tout son sens.

II est vrai que la Charte est un document suffisamment large pour qu'il ne soit pas impossible qu'une interprétation généreuse assure un jour une égalité sociale plus substantielle aux groupes dominés. De par sa logique même toutefois, elle reste un document irrémédiablement libéral qui n'a d'autre conséquence que de maintenir, voire de redéployer, les assises sociales et économiques des groupes dominants de la société canadienne.

\section{Libéralisme et postmodernité : jalons pour une interprétation}

Sous couvert d'un élargissement théorique de la sphère d'égalité et de justice sociales, l'enchâssement de la Charte

7. La Cour d'appel de la Nouvelle-Écosse a remédié au silence de la législation provinciale au sujet de l'assistance sociale aux pères célibataires en annulant l'avantage conféré en ce sens aux mères célibataires. En Colombie-Britannique, en réponse à une décision du tribunal désapprouvant l'octroi de prestations de bien-être social inférieures aux moins de 26 ans, le gouvernement provincial a tout simplement choisi de réduire les prestations pour toutes les catégories de bénéficiaires (Hutchinson et Petter, 1989, p. 293). Au cours des premières années d'entrée en vigueur de l'article 15 de la Charte, plusieurs actions ont été entreprises avec succès par des hommes qui remettaient en question des protections et des avantages législatifs favorisant les femmes, tels que la réforme de la loi sur le viol et les prestations d'assurance-chómage en cas de grossesse (Brodsky et Day, 1989, chapitre 3). 
des droits et libertés dans la Constitution concourt a la reproduction de rapports de force inégalitaires et à l'obsolescence de pratiques démocratico-populaires fondées sur la souveraineté parlementaire. Balise nouvelle en apparence, qui ne fait que reconduire dans leur intégralité et en les intensifiant les structures traditionnelles de l'inégalité et les rapports de force socio-économiques désavantageux pour les groupes dominés.

Voila qui résume le sens politique profond de la Charte et du type de société qu'elle enserre. Mais, partant, quel genre d'arrangement socio-institutionnel et, surtout, quel genre de dynamique sociopolitique cette situation héritée des années 80 permet-elle d'entrevoir au seuil du $X X 1^{\circ}$ siecle?

Monument de l'ambivalence érigée en système, la Charte est, dans sa facture, caractéristique de l'historicité propre du libéralisme canadien dont Gad Horowitz a dit qu'il présente "first its radical face and then its conservative face, urging reforms and warning against hasty, ill-considered change, calling for increased State responsibility, but stopping short of socialism openly, speaking for the common people, but preaching the solidarity of classes" (Horowitz, 1968, p. 34).

Mais le problème en est plus simplement un de forme, de style ou de tradition. II est plus vaste et plus dramatique a la fois, car il touche au cœur de l'intégrité paradigmatique du libéralisme même. Paradoxalement, la Charte incarne la faillite du libéralisme démocratique au Canada. L'avenir politique du pays s'articule autour de cette réalité et en dépend.

La Charte est tout a fait typique de la mouvance culturelle postmoderniste de notre fin de millénaire qui, ellemême, participe du mouvement de désorganisation/restructuration du capitalisme tant à l'échelle mondiale que domestique (Lash et Urry, 1987). Un univers de simulation, de simulacres où les symboles de la réalité prennent en quelque sorte le pas sur la réalité elle-même. La Charte est le surgeon d'un libéralisme arrivé à son point de non-retour, d'un libéralisme qui table sur son image de liberté et d'idéaux démocraticohumanitaires, mais qui, dans sa pratique, renvoie l'inverse de l'image qu'il projette - miroir déformant de l'hyperréalisme postmoderne. 
Le libéralisme canadien reprend en cela la posture schizophrène du libéralisme démocratique occidental à propos duquel Benjamin Barber a noté : «torn within and divided against itself, liberal democracy sets its means against its ends. Its tools of liberation become instruments of subjugation, while its individualist objectives become the agents of social disorder and anomie" (Barber, 1984, p. 14). Implosion du sens, dirait Baudrillard : le libéralisme canadien est attiré dans le vortex irrésistible d'une symbolique débridée où le contraire du vrai est le vrai, où les sous-entendus et les nondits font loi, où les perceptions, fondées ou non, façonnent le réel. Implosion du sens, mais aussi discours de la fausse conscience, avec son train de manipulations idéologiques servant à justifier et à faire accepter la position de force du dominant.

La Charte démontre combien les classes dominantes sont coincées entre la dégénérescence de l'hégémonie du projet socio-économique historique qu'elles défendent et l'impérative nécessité de résister aux assauts multiples montés contre elles. En tablant sur le discours des droits, on banalise les conflits qui traversent la société, on les sort de l'ordre du politique pour les intégrer à l'ordre du juridique. On calme la grogne. Le discours des droits apaise le mécontentement populaire en créant l'impression d'un élargissement de la démocratie, en créant la perception d'une citoyenneté plus générale, plus englobante. En réalité, il limite l'exercice de cette citoyenneté puisqu'il en confine la pratique au juridique. On a façonné de toutes pièces, à travers la Charte, l'illusion d'une plus grande égalité, d'une démocratie plus efficace alors qu'il n'en est rien (Banting et Simeon, 1983; Whitaker, 1983).

Les Canadiens ont maintenant une Constitution fondée sur la recherche de l'égalité sociale alors que cette égalité n'existe pas et ne peut exister parce que le libéralisme s'articule de manière presque viscérale à des structures sociales fondamentalement définies et déterminées par l'inégalité (Barber, 1984, p. 110). Triomphe de la raison cynique et du simulacre? On laissera a la théorie critique le soin de deviser sur cette attitude psychoculturelle typique, semble-t-il, de la postmoder- 
nité. Les conséquences $n^{\prime}$ en sont pas moins lourdes pour l'avenir du jeu politique canadien.

La Charte $n$ 'améliore en rien l'équilibre des forces sociales au Canada; elle renforce même, de par son application, les schémas traditionnels d'inégalité socio-économique. On pourrait conclure que l'hégémonie menacée est préservée. En réalité, la constitution et l'enchâssement de la Charte créent en termes politiques une situation potentiellement explosive. Dans la mesure où les classes et groupes historiquement dominants consolident leur position et leur pouvoir, dans la mesure où le fossé continue de s'agrandir entre privilégiés et défavorisés, entre possédants et démunis, entre régions riches et régions pauvres, les rapports de force qui jouent au désavantage des laissés pour compte de la société leur deviendront de plus en plus intolérables. D'autant plus que ces groupes défavorisés ou ceux qui prennent leur défense sont maintenant justifiés de remettre en question ces rapports de force. La Charte leur fournit le prétexte et les outils nécessaires à cette fin ou à tout le moins l'illusion que l'inégalité des rapports de force est inadmissible. Sur la base de cette illusion, on verra se multiplier les défis au code dominant d'interrelation sociale.

En soi, voilà qui devrait plutôt paraître réjouissant. Mais I'antinomie qui existe entre les larges droits théoriques, garantis par la Charte, et les droits réels et restreints dont l'application permet la jouissance pose un problème que la judiciarisation du politique écarte mais ne résoud pas : la profusion de droits non exercés auparavant crée sur les institutions politiques des pressions de plus en plus difficiles à gérer. La Charte laisse croire à ces groupes sociaux, historiquement contraints au silence politique, qu'ils ont désormais voix au chapitre. Bien qu'il leur soit encore difficile - et parfois impossible - de se faire entendre, ils chercheront de façon illusoire à affirmer ces droits que, sans réelle conviction, on leur promet.

$L^{\prime}$ ordre politique qui se dessine au Canada à l'aube du XXI" sieccle prend forme dans l'écart entre les promesses théoriques du renouveau qu'offre la Charte et la volonté non avouée de produire une réalité qui les nie. Il prend aussi forme dans 
l'écart entre ce qu'on dit vouloir et ce qu'on veut en fait. La politique de l'an 2000 au Canada émergera des solutions que I'on aura su trouver à cette contradiction. Ou elle sera la conséquence de l'absence de solutions.

En attendant, l'actualité nous offre un avant-goût du cheminement que nous aurons sans doute à faire. L'échec de l'accord du Lac Meech et la crise amérindienne sont symptomatiques. Les Québécois sont frustrés qu'on ne reconnaisse pas leurs demandes historiques minimales; le gouvernement central est dégoûté de ce que des intérêts régionalistes étroits aient fait avorter son grand plan de réconciliation nationale; les autochtones sont déterminés à faire respecter leurs revendications et droits ancestraux; la population blanche est outrée jusqu'au racisme et à l'intolérance d'avoir à faire les frais de 300 ans d'histoire dont elle ne se sent pas responsable. C'est donc un concert de voix discordantes qui s'élève, toutes ces voix se réclamant de la Constitution et étant aussi justifiées les unes que les autres de le faire.

En judiciarisant le politique, les autorités politiques ont abdiqué en partie leur responsabilité et laissent la société civile à elle-même. Les intérêts divergents restent sur leur position, irréconciliables. Le Canada est peut-être en train de s'engager dans une voie où il serait impossible de gouverner.

Le moment constitutionnel de 1982 a institué une mouvance nouvelle susceptible de reformuler la culture politique canadienne. Le discours des droits que propose implicitement la Charte à l'imaginaire sociopolitique impose un nouveau code d'interaction entre les citoyens eux-mêmes comme entre les citoyens et l'État. Ce code, à tort ou à raison, permet a chacun de croire en la rectitude de ses positions; à terme, il menace d'estomper la frontière imprécise entre la liberté de I'Un et la liberté de l'Autre.

C'est à ce point que les choses se compliquent et que plane la menace d'une société ingouvernable. Chacun, soit à travers son individualité propre, soit à travers le groupe auquel il s'identifie, est justifié de réclamer les mêmes avantages que l'autre. Or, malgré le discours spécieux de la Charte, cela est impossible à obtenir en raison de l'inégalité intrinseque sur laquelle repose la logique de la société canadienne. Cette 
logique, la judiciarisation du politique et la Charte elle-même ne l'altéreront pas d'un iota.

La Charte a permis que se manifestent de nouveaux sujets politiques, de nouvelles sensibilités identitaires. La Constitution est devenue une arène au sein de laquelle les groupes sociaux définis en termes linguistiques, ethniques, culturels, raciaux et sexuels luttent les uns avec les autres pour la reconnaissance de leur statut propre et leur libération socioéconomique. Les débats publics préalables au rapatriement de la Constitution avaient donné à ces groupes l'impression qu'il était possible d'influencer avec succès le processus décisionnel. A cette époque, les groupes de pression, préoccupés par les nouvelles balises constitutionnelles dont on allait doter le pays, ont directement participé à l'exercice de redéfinition constitutionnelle et en ont, à plusieurs égards, façonné le résultat (Williams, 1985). Pourtant, le processus devant mener à l'accord du Lac Meech et à son rejet montrait, à peine quelques années plus tard, que le Gouvernement n'entendait pas faire de cette participation une tradition. Du début à la fin, le sort de l'Accord a été réglé par onze hommes, fort peu représentatifs somme toute des voix qui s'étaient préalablement fait entendre : ils les auront d'emblée mises au silence par leurs tractations à huis clos. L'épisode du lac Meech prouve sans ambages que, si la Charte a permis que s'expriment de nouvelles sensibilités identitaires, le système politique, lui, n'est pas vraiment disposé à leur faire la place qui leur revient (Cairns, 1988). Par la Charte, on a posé les éléments d'une dynamique nouvelle à condition, semble-t-il, que le mouvement $n$ 'en soit jamais actionné.

Il est possible d'envisager au moins trois points de rupture dans l'ordre politique canadien des années à venir. Premièrement, la multiplicité des intérêts divergents et contradictoires laisse entrevoir l'exacerbation des tensions entre les groupes dans la lutte pour l'affirmation des principes d'égalité, de justice sociale et de respect des particularismes identitaires. La tendance au retrait volontaire du pouvoir politique augure mal. Impuissant ou non disposé à mobiliser la sphère publique pour résoudre ces tensions, l'État pourrait être appelé a déployer ses appareils répressifs et coercitifs. L'histoire du 
pays et, plus récemment, la crise d'Oka nous apprennent que I'État canadien n'est pas insensible à cette tentation.

Le deuxième point de rupture s'objectivera dans une probable crise de légitimité de l'État, de ses institutions et des agents sociaux qui en dépendent. Tant que le système politique continuera de repousser les sans-voix à la marge alors qu'il leur promet la tribune, il faudra inévitablement $s^{\prime}$ attendre à une crise de confiance face au système lui-même et, éventuellement, à une remise en question de la légitimité des fondements de l'État. Le Québec, on le sait, s'est aventuré dans cette voie depuis longtemps, mais de manière mitigée jusqu'à maintenant, participant de plein gré à et de l'État canadien. D'autres, Mowhaks en tête, ont commencé à signifier sans équivoque leur distanciation socio-institutionnelle de l'État canadien.

Enfin, il est possible d'imaginer un troisième point de rupture sur lequel se jouera aussi l'avenir politique du Canada. C'est la rupture du libéralisme lui-même. Son insistance sur l'individualisme, son déni du pouvoir et de la contrainte au sein de la société, sa glorification du marché comme principe d'interaction sociale sont des notions qui le posent pour ainsi dire en contradiction théorique avec la réalité sociale de la fin du $X X^{*}$ siècle. Dans les faits, cette réalité opère à contrecourant des objectifs constitutifs du libéralisme. Le marché n'est pas autoréglé et ne pourrait fonctionner convenablement sans l'appui de l'État. Les tendances lourdes de la société civile démontrent l'incontournable prégnance du pouvoir et de son exercice inégalitaire. Les problématiques en apparence privées apparaissent toujours plus indissociables de problématiques publiques. Bref, la primauté du collectif s'impose constamment à l'individuel, celle du social à l'économie.

L'après-guerre et la montée de l'État-providence ont consacré la fatalité historique de cette réalité. Ce n'est qu'en réaction à ce qui est apparu comme les excès du collectif que le libéralisme s'est manifesté de nouveau dans son individualisme radical - retour de l'histoire. Quoi qu'il en soit, cette volonté presque obsessionnelle de reconduire le libéralisme dans ses oripeaux classiques demeure "contre nature». 
II y a près de 50 ans maintenant, Karl Polanyi a démontré dans son ouvrage magistral The Great Transformation (1944) que la société capitaliste s'objective historiquement en un double mouvement qui, de la primauté du marché, ramène toujours la société vers le social : action spontanée qui vise à protéger la société contre les excès du marché et d'un individualisme débridé. II est très probable que l'avenir du Canada prendra forme dans la résolution de cette antinomie. Le défi de l'an 2000 sera de trouver l'équilibre entre les droits de l'individu et les nécessaires responsabilités sociales et communautaires. 


\section{Bibliographie}

BANTING, K. et SIMEON, R., éds., And No One Cheered : Federalism, Democracy and the Constitution Act, Toronto, Methuen, 1983.

BARBER, B., Strong Democracy. Participatory Democracy for a New Age, Los Angeles, University of California Press, 1984.

BEATTY, D. M., Putting the Charter to Work. Designing a Constitutional Labour Code, Montréal, McGill-Queen's University Press, 1987.

BEAUDOIN, G. et RATUSHNY, E., éds., The Canadian Charter of Rights and Freedoms, $2^{\circ}$ éd., Toronto, Carswell, 1989.

BOISMENU, G., "L'État et la régulation du rapport salarial depuis 1945", dans BOISMENU, G. et DRACHE, D., éds., Politique et régulation. Modèle de développement et trajectoire canadienne, Montréal et Paris, Méridien et L'Harmattan, 1990, p. 155-204.

BRODSKY, G. et DAY, S., La Charte canadienne et les droits des femmes. Progres ou recul?, Ottawa, Conseil consultatif canadien sur la situation de la femme, 1989.

CAIRNS, A.C., "Citizens (Outsiders) and Government (Insiders) in Constitution-Making. The Case of Meech Lake», Canadian Public Policy, vol. 14, 1988, p. S121-S145.

DRACHE, D. et GLASBEEK, H.J., "Le néo-fordisme. L'offensive du capital, l'alternative des travailleurs» dans BOISMENU G. et DRACHE D., éds., Politique et régulation. Modèle de développement et trajectoire canadienne, Montréal et Paris, Méridien et L'Harmattan, 1990, p. 237-274.

ELKINS, D., "Facing Our Destiny : Rights and Canadian Distinctiveness", Canadian Journal of Political Science, vol. $22, n^{\circ} 4$, décembre 1989, p. 699-716. 
FINLAYSON, J., «La politique économique internationale du Canada. Contexte, problèmes et examen de quelques analyses récentes", dans STAIRS D. et WINHAM W., éds., Quelques problèmes concernant l'élaboration de la politique économique extérieure, Toronto, University of Toronto Press, 1985.

GOUVERNEMENT DU CANADA, Une étude de la politique commerciale canadienne, Ottawa, Approvisionnements et Services, 1983.

GREENE, I., The Charter of Rights, Toronto, Lorimer, 1989. HOROWITZ, G., Canadian Labour in Politics, Toronto, University of Toronto Press, 1968.

HOULE, F., «L'État canadien et le capitalisme mondial : stratégies d'insertion", Revue canadienne de science politique, vol. $20, n^{\circ} 3$, septembre 1987, p. 467-500.

HUNTER, A., Class Tells. On Social Inequality in Canada, $2^{\circ}$ éd., Toronto, Butterworths, 1986.

HUTCHINSON, A.C. et PETTER, A., «Private Rights/Public Wrongs : The Liberal Lie of the Charter", University of Toronto Law Journal, vol. 38, 1988, p. 278-297.

JENSON, J., "Different" but not "Exceptional". Canada's Permeable Fordism», Canadian Review of Sociology and Anthropology, vol. 26, $\mathrm{n}^{\circ} 1,1989$, p. 69-94.

KNOPFF, R. et MORTON, R.F., "Nation-Building and the Canadian Charter of Rights and Freedoms», dans CAIRNS A. et WILLIAMS C., éds., Constitutionalism, Citizenship and Society in Canada, Toronto, University of Toronto Press, 1985, p. 133-182.

LANGILLE, D., "The Business Council on National Issues and the State", Studies in Political Economy, vol. 24, automne 1987, p. 41-86.

LASH, S. et URRY, J., The End of Organized Capitalism, Cambridge, Polity Press, 1987.

MANDEL, M., The Charter of Rights and the Legalization of Politics in Canada, Toronto, Wall and Thompson, 1989.

MAXWELL, J. et PESTIEAU, C., Economic Realities of Contemporary Confederation, Montréal, C.D. Howe Institute, 1980. 
MILNE, D., «Politics and the Constitution», dans GAGNON A.G. et BICKERTON, J.P., éds., Canadian Politics. An Introduction to the Discipline, Peterborough, Broadview Press, 1990, p. 206-221.

MILNE, D., The Canadian Constitution. From Patriation to Meech Lake, 2 ed., Toronto, Lorimer, 1989.

MONAHAN, P., Politics and the Constitution. The Charter, Federalism and the Supreme Court of Canada, Toronto, Carswell, 1987.

MONAHAN, P. et PETTER, A., «Development in Constitutional Law : the 1985-1986 Term», Supreme Court Law Review, vol. 9, 1987, p. 69-143.

MORTON, F.L., "The Political Impact of the Canadian Charter of Rights and Freedoms", Canadian Journal of Political Science, vol. 20, $\mathrm{n}^{\circ} 1$, mars 1987, p. 31-55.

PETTER, A., "Canada's Charter Flight : Soaring Backwards in to the Future», Journal of Law and Society, vol. 16, $n^{\circ} 2$, été 1989, p. 151-165.

PETTER, A., "Immaculate Deception : The Charter Hidden Agenda", The Advocate, vol. 45, novembre 1987, p. 857866.

PETTER, A., «The Politics of the Charter», The Supreme Court Law Review, vol. 8, 1986, p. 473-505.

POLANYI, K., The Great Transformation, Boston, Beacon Press, 1944.

ROMANOW, R., WHYTE, J. et LEESON, H., Canada... Notwithstanding. The Making of the Constitution, 19761982, Toronto, Carswell, 1984.

RUSSELL, P., "The Charter and the Future of Canadian Politics» dans GAGNON, A.-G. et BICKERTON, J.P., éds., Canadian Politics. An Introduction to the Discipline, Peterborough, Broadview Press, 1990, p. 246-262.

RUSSELL, P., "The Effect of a Charter of Rights on the PolicyMaking Role of Canadian Courts", Canadian Public Administration, vol. 25, $n^{\circ} 1$, printemps 1982, p. 1-33. RUSSELL, P., «The First Three Years in Charterland", Canadian Public Administration, vol. $28, \mathrm{n}^{\circ} 3$, automne 1985, p. 367-396. 
RUSSELL, P., "The Paradox of Judicial Power", Queen's Law Journal, vol. $12, n^{\circ} 3,1987$, p. 421-437.

TARNOPOLSKY, W., "The Constitution and Human Rights", dans BANTING, K. et SIMEON, R., éds., And no One Cheered. Federalism, Democracy and the The Constitution Act, Toronto, Methuen, 1983, p. 261-279.

VANDYCKE, R., "Les droits de l'homme et leurs modes d'emploi : a propos de la charte constitutionnelle de $1982 "$, Sociologie et sociétés, vol. 18, $n^{\circ} 1$, avril 1986 , p. 139-151.

WHITAKER, R., "Democracy and the Canadian Constitution", dans BANTING, K. et SIMEON, R., éds., And no One Cheered. Federalism, Democracy and the Constitution Act, Toronto, Methuen, 1983, p. 240-260.

WILLIAMS, C., "The Changing Nature of Citizen Rights", dans CAIRNS, A. et WILLIAMS, C., Constitutionalism, Citizenship and Society in Canada, Toronto, University of Toronto Press, 1985.

WOLFE, D., "The Canadian State in Comparative Perspective", dans Canadian Review of Sociology and Anthropology, vol. 26, nं 1, 1989, p. 95-126.

WOLFE, D., "The Rise and Demise of the Keynesian Era in Canada : Economic Policy, 1930-1982», dans CROSS, M. et KEALEY, G., éds., Modern Canada, 1930-1980, Toronto, McClelland and Stewart, 1984. 